\title{
Detecting COVID-19 at the bedside
}

\author{
Ursula Hofer, Andrea Du Toit and Ashley York
}

Scientists around the world have risen to the challenges posed by COVID-19 by rapidly transforming their laboratories and refocusing their research efforts.

CSAMBA ...
increased hospital
efficiency and
safety by detecting
new infections in
hospital rapidly
and by freeing up
isolation rooms

At Nature Reviews Microbiology, we have been inspired by how the scientific community have redirected their research efforts to help tackle the COVID-19 pandemic. This global effort has been instrumental in helping us to understand the epidemiology, dynamics and biological features of the new coronavirus and for paving the way for the development of therapeutics and vaccines. In the second of a new series of Feature articles to highlight these efforts, we interviewed Ravi Gupta (professor), Steve Kemp (postdoctoral fellow), Dami Collier (PhD student) and Bo Meng (Research Associate) at The Cambridge Institute for Therapeutic Immunology and Infectious Diseases (CITIID).

\section{How has your institute adapted to tackling COVID-19, and what is the impact of this work?}

R.G. CITIID is part of the Department of Medicine at the University of Cambridge. It opened just before the COVID-19 pandemic with the largest academic biosafety level 3 (BSL3) lab in the UK, with a remit of doing cutting edge basic and translational science in infection and immunity. While the rest of the campus closed down during the first wave, we remained open in order to rapidly respond. This was what CITIID was designed for in many respects. We realized there was a need for rapid nucleic acid testing at the hospital, so we worked with colleagues in industry to bring a near patient isothermal platform for nucleic acid detection (a simple amplification-based assay (SAMBA)) to the clinic. We did a large study to show that actual turnaround time was tenfold faster with SAMBA, and it increased hospital efficiency and safety by detecting new infections in hospital rapidly and by freeing up isolation rooms. We also developed a lab serum neutralization

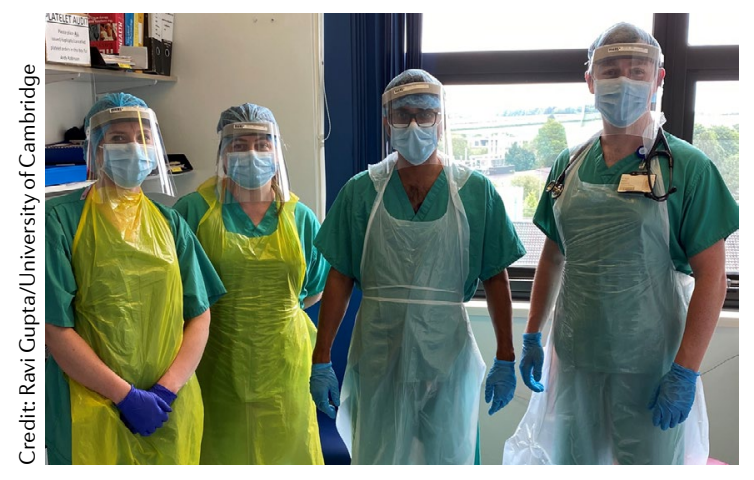

test that we used to detect antibody responses in clinical samples, and published a study showing that we could combine antibody and nucleic acid rapid tests to increase diagnostic accuracy of COVID-19, and to detect the newly emergent D614G spike mutant. Other work in the institute has included mass staff testing, defining immune responses to SARS-CoV-2, innate immune responses in myeloid cells to SARS-CoV-2 and use of next-generation sequencing to detect transmission networks in real time.

S.K. I used to work on HIV. As news quickly spread of the novel SARS-CoV-2, almost all of us quickly switched to working towards a better understanding of that virus instead. It is exciting to see many different colleagues from different backgrounds (such as evolutionary biologists, bioinformaticians and virologists) come together. I was pleased to note that many of the skills I learnt while analysing HIV (from my previous work) have been directly applicable to the analysis of SARS-CoV-2. Specifically, we have been fortunate to have access to patients with numerous sequential COVID-19-positive samples, which have all been sequenced using both Illumina and Nanopore technologies. As such, we have been able to examine intra-host diversity in patients with numerous sequential samples and use phylogenetic methods to show changes in the virus over time. As we continue to characterize such changes, we anticipate that this work will prove valuable in determining how various treatments put pressure on the virus and force it to mutate.

D.C. I watched as the pandemic swept across Asia, into Europe and then arrived at our shores. The direct impact was felt immediately, and the challenges of testing were acute in our hospital, Cambridge University Hospitals NHS Trust. I paused my PhD in the Gupta lab at CITIID to focus on diagnostic accuracy studies of rapid diagnostic tests to detect SAR-CoV-2 in hospitals. I have background training in epidemiology and was pleased to be able to use these skills in the COVID-19 response. Knowledge about the virus is growing exponentially, and there is still so much to discover. It has been really exciting being at the forefront this.

B.M. Our institute is relatively new. Sitting at the centre of its research programmes are immunological 
interventions and infectious diseases. All the research programmes were shut down, and priorities were given to COVID-19 related research. The management of our institute quickly set up research programmes ranging from COVID-19 diagnostics to potential therapeutics.

\section{How has your daily work life changed?}

R.G. We have become single minded about addressing multiple aspects of COVID-19 based on our expertise, both basic science and clinical. Luckily I live 10 minutes walk from my lab and the hospital, and the warm dry summer helped us all through the lockdown. Cambridge has great outdoor activities that helped us relax during this stressful time. Previously I used to travel to South Africa where I have a faculty position at Africa Health Research Institute, which is an HIV/TB research institute funded by the Wellcome Trust. This and other meeting-related travel has stopped, though for good reason. I certainly miss those face-to-face scientific interactions.

S.K. Prior to the COVID-19 outbreak, I did a lot of international travel, meeting many new people, presenting my work at conferences and collaborating with overseas colleagues. Now, I tend to spend a lot of time at my computer in virtual meetings, trying to co-ordinate various projects, students and lab meetings between different groups.

D.C. During this time, I worked very long hours and missed out on that bonding time in lockdown that some families have described. I find that I'm able to work more flexibly and work from home as well. I'm able to access more learning opportunities and talks through online platforms that I would not otherwise have been able to attend if it required travel.

B.M. I was among a small group of people who were privileged to be still able to come to work during the lockdown. The adaptation, however, was not straightforward as managing to get the work efficiently done while bearing the responsibility of home schooling proved to be challenging. On top of that, we had a visitor staying with us from overseas before the lockdown, and the knock-on effect of lockdown in the aviation industry meant he had to stay with us for a prolonged period. Given the work I was doing, and him being in an at-risk group, I was particularly cautious with my hygiene when I got back home.

\section{What challenges are you facing right now, and what challenges do you anticipate?}

R.G. I would have very much liked to conduct some of the COVID-19 testing work in South Africa, but for various reasons we did this all in the UK. We have been relatively fortunate in Cambridge as the University has been supportive of our work. Staff testing here in our building would be desirable but in the current climate difficult.

S.K. Working from home has been challenging to adapt to as I do genuinely miss interacting with others in the workplace environment, face-to-face. Having a morning briefing with our cups of coffee helps to get everybody focused for the day ahead; working from home definitely has its downsides, as there is temptation to procrastinate and enjoy the garden instead of working!

D.C. Certain core facilities were redirected towards the COVID-19 response, such as the BSL3 labs. It's been difficult to get the usual HIV BSL3 work up and running alongside this.

B.M. Being a virologist, there is a huge sense of wanting to work even harder to help alleviate the situation that this virus has caused and is still causing adversely on our daily lives worldwide. As a result of this sense of urgency, things may be stretched a bit thin, not only on the existing research projects but also on COVID-19 research. I found fine-tuning the balance of these two areas is challenging.

What were you working on before the COVID-19 pandemic, and how is this work being impacted?

R.G. Our work on drug-resistant HIV and reservoirs of HIV in the brain being conducted in South Africa has halted largely, though we have collaborations in Uganda and Nigeria that are ongoing. Here in the UK, we stopped our lab work on HIV drug resistance but continued our HIV genetics and phylogenetic work, but we recently restarted our lab work. Our studies extend to epidemiology, and we have been able to continue analysis and publication of work on HIV and mortality in Africa. We also have a programme of work on macrophages, cell cycle regulation and innate immune responses that is highly relevant for COVID-19 given the uncontrolled inflammation that occurs, reminiscent of macrophage activation syndrome. This work has continued throughout in an effort to understand how hyperinflammation occurs and can be treated.

S.K. I have previously worked in South Africa (as well as London and Cambridge), assessing drug-resistance mutations and transmission of drug-resistant virus between people, in HIV-infected individuals. The majority of that work ceased during the initial months of the COVID-19 pandemic. As the majority of my work is computational in nature, I have been fortunate to continue working on both HIV and SARS-CoV-2.

D.C. I was doing a $\mathrm{PhD}$ on $\mathrm{HIV}$ and the impact on the brain - in particular studying HIV genetics in blood versus the cerebrospinal fluid, as well as phenotypic properties of these viruses. It has been almost impossible to leave my COVID-19 work and return to my $\mathrm{PhD}$ given that there are so many unanswered questions that require our clinical and virology skills.

B.M. I was working on how oxygen tension affects virus infection in mammalian cells. During the lockdown, this research was largely suspended giving way to COVID-19 research on the effects of repurposed drugs on immune responses in primary cells.

Ursula Hofer, Andrea Du Toit and Ashley York

Nature Reviews Microbiology.

e-mail:nrmicro@nature.com

https://doi.org/10.1038/s41579-020-00464-w 